Research Article

\title{
The Derivative of Tripterygium wilfordii Hook F-Kunxian Capsule, Attenuated Rheumatoid Arthritis: A Systematic Review and Meta-Analysis
}

\author{
Ya-Fei Liu $\mathbb{D}^{1},{ }^{1}$ Zhe Zhang, ${ }^{1}$ Jun-Jun Zhang, ${ }^{1}$ Zhe Chen $\mathbb{D}^{2}{ }^{2}$ Sheng-Hao Tu $\left(\mathbb{D},{ }^{2}\right.$ \\ and Guo-Lan Xing ${ }^{1}$ \\ ${ }^{1}$ Department of Nephrology, The First Affiliated Hospital of Zhengzhou University, 1 Jianshe East Road, Zhengzhou, \\ Henan 450052, China \\ ${ }^{2}$ Institute of Integrated Traditional Chinese and Western Medicine, Tongji Hospital, Tongji Medical College, \\ Huazhong University of Science and Technology, 1095 Jiefang Avenue, Wuhan, Hubei 430030, China
}

Correspondence should be addressed to Ya-Fei Liu; yafeiliutjh@gmail.com

Received 7 April 2020; Revised 29 June 2020; Accepted 17 July 2020; Published 12 August 2020

Guest Editor: Lei Xu

Copyright (c) 2020 Ya-Fei Liu et al. This is an open access article distributed under the Creative Commons Attribution License, which permits unrestricted use, distribution, and reproduction in any medium, provided the original work is properly cited.

The study aimed to explore the efficacy and safety of Kunxian Capsule (KXC) in the treatment of rheumatoid arthritis (RA). The randomized controlled trials (RCTs) comparing the effects of KXC in patients with RA were included in this study. Weighted mean differences (MDs) were calculated for net changes by employing Review Manager meta-analysis software. Nine RCTs were included in the systematic review with a total of 747 patients. The overall effects showed that KXC alone or combined with diseasemodifying antirheumatic and drugs decreased tender joint counts $(P=0.02, \mathrm{MD}=-1.07,95 \% \mathrm{CI}:-1.95$ to -0.18$)$, shortened duration of morning stiffness $(P<0.0001, \mathrm{MD}=-9.01,95 \% \mathrm{CI}:-13.08$ to -4.93$)$, lowered erythrocyte sedimentation rate $(P<0.00001, \mathrm{MD}=-5.27,95 \% \mathrm{CI}:-6.78$ to -3.77$)$, and reduced $\mathrm{C}$-reactive protein $(P<0.0001, \mathrm{MD}=-5.04,95 \% \mathrm{CI}:-7.28$ to $-2.80)$. The most common adverse events were gastrointestinal disturbances and abnormal liver function. These results suggest that KXC is likely to be a more effective and safe candidate for treating RA compared with conventional therapies.

\section{Introduction}

Rheumatoid arthritis (RA) is an autoimmune disease inducing synovial inflammation, which is characterized by tenderness, swelling, morning stiffness, joint destruction, and deformity $[1,2]$. The age-standardized prevalence and incidence rates are increasing, especially in countries such as Canada, Paraguay, and Guatemala. To reduce the ongoing burden of this condition, early diagnosis and treatment of RA are vital, especially among female patients [3].

The conventional therapies include nonsteroidal antiinflammatory drugs (NSAIDs), glucocorticoids, and diseasemodifying antirheumatic and drugs (DMARDs) $[4,5]$. With the exploration of molecular mechanisms, biological agents have been wildly applied in the treatment of RA $[6,7]$. However, owing to the adverse effects of conventional medications and high financial stress of biologicals [8], more effective and safe medications for RA are needed to be explored.

Tripterygium wilfordii Hook F (TWHF) has been applied in the treatment of RA in China for decades [9]. The previous research showed that TWHF could be as effective as synthetic DMARDs in the treatment of RA and the common adverse effects (AEs) were gastrointestinal discomfort, menstruation disorders, and amenorrhea [10]. Kunxian Capsule (KXC) is a derivative of TWHF and has little liver injury and reproductive toxics compared with TWHF [11]. Meanwhile, KXC has been employed in treating RA in clinical trials for a long time [12-14]. However, most of these clinical data origins from uncontrolled clinical trials or retrospective reports, and few multicenter clinical trials have been performed to verify the effects of $\mathrm{KXC}$ in the treatment 
of RA. Therefore, the scientific evidence confirming the efficacy and safety of KXC in treating RA remains unclear. Given these uncertainties, it is essential to perform a quantitative meta-analysis of the efficacy and safety of KXC in patients with RA. Consequently, we carried out the metaanalysis and systematic review.

\section{Materials and Methods}

The Preferred Reporting Items for Systemic Review and Meta-Analyses (PRISMA) statement [15] was applied to design and report the study.

2.1. Search Strategy. The following English databases were retrieved to confirm trials: PubMed, the Cochrane Library, and Clinical Trials.gov. In addition, the Chinese databases, such as the CNKI Database, Wanfang Database, VIP Database, CBM Database, and Chinese Clinical Trial Register were searched. All the databases were retrieved from their available dates of inception to the latest issue (June 2019).

Different search strategies were combined as follows. For the English databases, free text terms were employed, such as "kunxian capsule" and "rheumatoid arthritis" or "RA". For the Chinese databases, free text terms were applied, such as "kunxian jiaonang" (which means Kunxian Capsule in Chinese) and "lei feng shi guan jie yan" (which means rheumatoid arthritis in Chinese). A filter for clinical trials was selected for English databases. To include an adequate number of trials, the reference lists of pertinent publications were also retrieved to identify additional studies.

2.2. Selection Criteria. Randomized controlled trials (RCTs) were included irrespective of blinding, publication status, or language. Studies were selected for reanalysis if they met the following criteria: (1) patients enrolled were diagnosed with RA, according to the 1987 or 2010 RA guidelines of the American Rheumatology Association; (2) in the intervention group, the participants took KXC alone or with other DMARDs; (3) in the control group, the participants took DMARDs; (4) duration was at least 12 weeks; and (5) outcomes were available.

Case reports, reviews, in vitro experiments, retrospective studies, or studies without control groups were excluded. For repeat studies, the authors were contacted to illuminate any ambiguities. The RCTs that lacked crucial data to allow for the calculation of net changes in outcomes and their variances from baseline to the endpoint were also excluded from the research. The literature was selected by two reviewers (YF Liu and Z Zhang) independently. The flowchart of the study selection has been generated in accordance with the PRISMA statement.

2.3. Data Extraction and Management. The data were extracted by two independent reviewers (Zhang and Chen), and contradictions were resolved by consensus or were judged by another reviewer.
The studies' quality was evaluated according to the Cochrane handbook 5.3. The risk of bias of the included trials was evaluated by the following items: (A) random sequence generation (selection bias); (B) allocation concealment (selection bias); (C) blinding of participants and personnel (performance bias); (D) blinding of outcome assessment (detection bias); (E) incomplete outcome data (attrition bias); (F) selective reporting (reporting bias); and (G) other bias.

The primary outcomes were tender joint count (TJC), swollen joint count (SJC), and duration of morning stiffness (DMS). The secondary outcomes included erythrocyte sedimentation rate (ESR), C-reactive protein (CRP), rheumatoid factor (RF), and anticyclic citrullinated peptide (CCP). Adverse events were also collected from the studies. For the trials that applied a three-armed group design, the outcomes of the groups were extracted if they satisfied the inclusion criteria. In terms of vagueness or absence in the publications of the outcomes, the authors were connected and related data has been extracted by consensus if the authors were unavailable.

2.4. Data Synthesis and Analysis. The effects of KXC on patients with RA were calculated as differences between the KXC group and the control group, utilizing the Review Manager meta-analysis software, version 5.3. To guarantee the credibility of the results, the net changes in all the outcomes were analyzed as the mean differences (KXC minus control) in changes (endpoint minus baseline) for parallel trials. The weighted mean differences and $95 \%$ confidence intervals (CIs) were calculated for continuous data. Heterogeneity was estimated through the chi-square test and Higgins $I^{2}$ test. A fixed-effect model was applied when the studies were sufficiently alike $(P>0.10)$; otherwise, a random-effects model was applied. For the subgroup with high heterogeneity, a random-effects model was performed. A Z score was calculated to determine the overall effect, with a significance set at $P<0.05$. Publication bias was analyzed by funnel plot if the number of the included studies $\geq 10$.

Two subgroup analyses were performed to diminish the clinical heterogeneity: KXC compared with DMARDs and KXC plus DMARDs compared with DMARDs.

\section{Results}

3.1. Study Selection. The process of study selection was shown in Figure 1. After filtering, 5 animal experiments, 6 reviews, 4 studies without sufficient data, 2 studies with duplicated data, and 4 studies whose control intervention did not meet the inclusion criteria were excluded. According to the selection criteria defined in the Materials and Methods, 9 RCTs were included in the meta-analysis. The three-armed group design was applied in two of the studies. To prevent sample duplication, the data of the two groups were included [16, 17]. In one study, there were three groups: leflunomide alone, leflunomide plus $\mathrm{KXC}$, and leflunomide plus methotrexate [16]. The data of leflunomide plus KXC versus leflunomide alone was extracted [16]. One study reported that KXC monotherapy was compared with methotrexate monotherapy or KXC plus methotrexate [17]. The data of KXC 


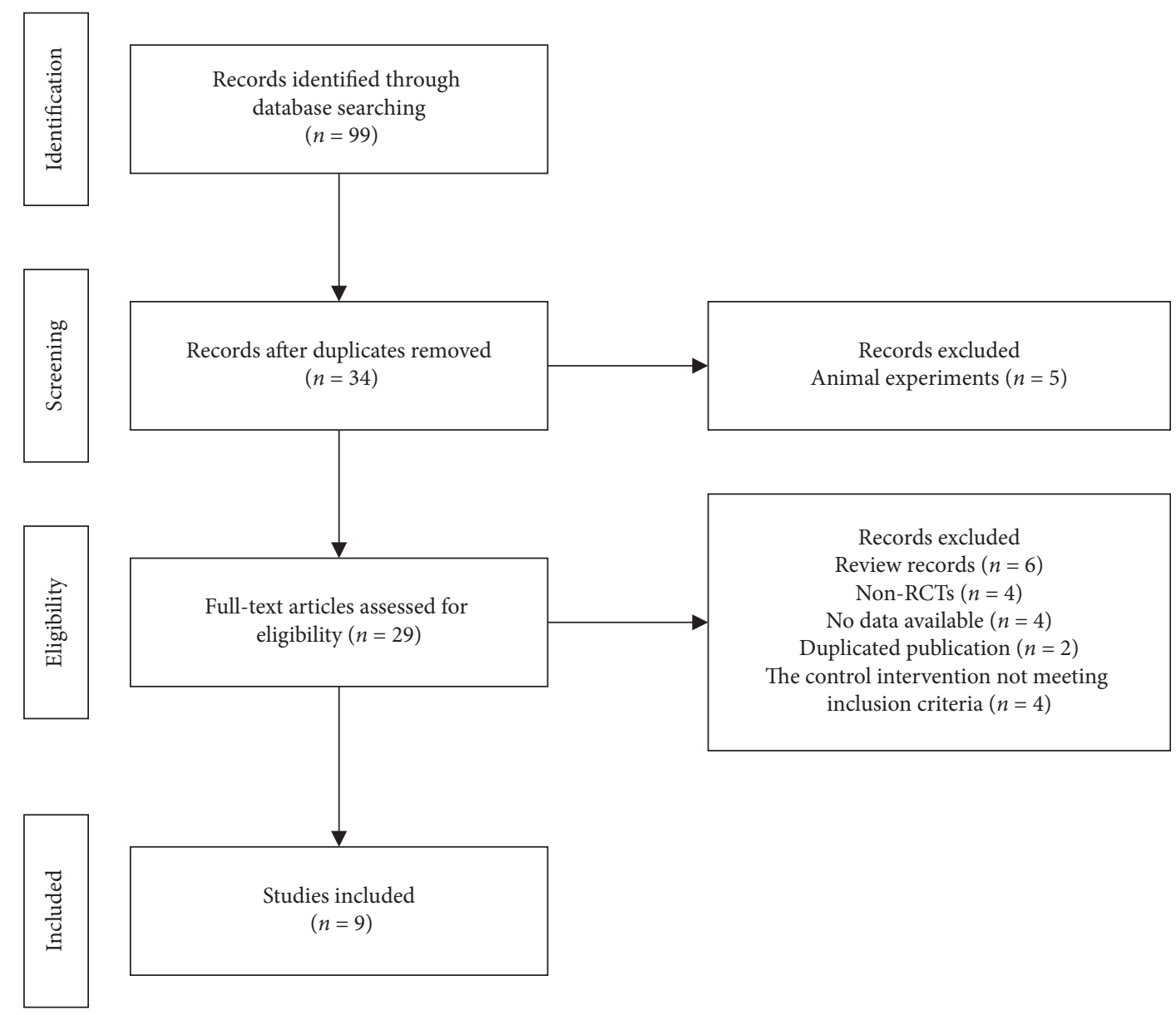

Figure 1: Flowchart of study selection.

monotherapy versus methotrexate monotherapy was extracted [17]. In the KXC monotherapy subgroup, three studies were included [17-19]. In the combined therapy subgroup, six studies were included [16, 20-24]. The characteristics of the studies were indicated in Table 1. Together, 747 RA patients were included in the study.

3.2. Study Descriptions. The included studies were published as full texts between 2010 and 2019. All the RCTs were performed in China. All the studies were published in Chinese. Eight studies were conducted as single-center trials, and only one study was a multicenter trial [17].

3.3. Interventions and Controls. Three studies compared KXC with DMARDs (methotrexate or leflunomide) [17-19]. Six trials compared a cointervention of KXC and DMARDs (methotrexate, or leflunomide, or hydroxychloroquine) with DMARDs [16, 20-24]. NSAIDs were added to the control group in two studies [20,21]. Different doses of KXC were administered in these studies. The KXC administration ranged from $0.9 \mathrm{~g}$ to $1.8 \mathrm{~g}$ per day. The total daily KXC was divided into two to three doses. The methotrexate administration ranged from $7.5 \mathrm{mg}$ to $15 \mathrm{mg}$ per week.
Except for one study with a duration of six months [19], all the other studies were 12 or 24 weeks. The outcomes were measured at 4 weeks and 12 weeks in two studies, and the outcomes of 12 weeks were extracted $[17,18]$. The outcomes were determined at 4 weeks, 8 weeks, and 12 weeks in one study, and the results of 12 weeks were extracted [22]. The outcomes were detected at 4 weeks, 8 weeks, 12 weeks, and 24 weeks in one study, and the results of 24 weeks were extracted [16].

3.4. Quality of the Included Studies. As indicated in Figure 2, all the included trials were of low quality due to unclear randomization, deficient allocation concealment, inadequate blinding, and undescribed withdrawals and dropouts.

3.5. Publication Bias. As the number of included studies $<10$, the funnel plot was not conducted to estimate the publication bias.

\subsection{Effects of Interventions}

3.6.1. The Outcome of TJC. As illustrated in Figure 3, only one study compared KXC with DMARDs in terms of TJC (involving 159 subjects), and there was no significant 
TABLE 1: Clinical and demographic characteristics of patients with rheumatoid arthritis.

\begin{tabular}{|c|c|c|c|c|c|c|c|c|}
\hline \multirow[t]{2}{*}{ Study } & \multicolumn{2}{|c|}{$\begin{array}{l}\text { Number of participants } \\
\text { (male/female) }\end{array}$} & \multicolumn{2}{|c|}{ Age (years) } & \multicolumn{2}{|c|}{ Intervention } & \multirow[t]{2}{*}{ Duration } & \multirow[t]{2}{*}{ Outcomes } \\
\hline & Experimental & Control & Experimental & Control & Experimental & Control & & \\
\hline Ji [20] & $30(3 / 27)$ & $30(5 / 25)$ & $47.93 \pm 11.57$ & $52.30 \pm 15.84$ & $\begin{array}{c}\text { KXC } 0.6 \mathrm{~g} \\
\text { bid + control }\end{array}$ & MTX $10 \mathrm{mg}$ qw, NSAIDs & $12 \mathrm{w}$ & (1) (2) (3) (4) (5) (6) \\
\hline Jia [22] & $30(9 / 21)$ & $30(8 / 22)$ & $22-70$ & $20-70$ & $\begin{array}{l}\text { KXC } 0.6 \mathrm{~g} \\
\text { tid + control }\end{array}$ & $\begin{array}{l}\text { MTX } 10-15 \mathrm{mg} \text { qw, folic } \\
\text { acid } 10 \mathrm{mg} \mathrm{qw}\end{array}$ & $12 \mathrm{w}$ & (1) (2) (3) (4) (5) (6) (7) \\
\hline Lu et al. [23] & $20(7 / 13)$ & $20(8 / 12)$ & $60-73(67)$ & $61-72(66)$ & $\begin{array}{c}\text { KXC } 0.3-0.6 \mathrm{~g} \\
\text { tid + MTX } 7.5 \mathrm{mg}\end{array}$ & MTX 7.5-15 mg qw & $24 \mathrm{w}$ & (1) (2) (3) (4) (5) \\
\hline $\begin{array}{l}\text { Quan and } \\
\text { Wang [24] }\end{array}$ & 78 & 78 & $54.29 \pm 11.08$ & $56.04 \pm 11.42$ & $\begin{array}{c}\text { KXC } 0.3-0.6 \mathrm{~g} \\
\text { tid + control }\end{array}$ & HCQ $0.2 \mathrm{~g}$ bid & $24 \mathrm{w}$ & (4) (5) (6) (7) \\
\hline $\begin{array}{l}\text { Zhang and } \\
\text { Zhang [21] }\end{array}$ & $36(13 / 23)$ & $36(15 / 21)$ & $45.6 \pm 10.2$ & $46.2 \pm 9.8$ & KXC 0.3 tid + control & $\begin{array}{l}\text { MTX } 10 \mathrm{mg} \\
\text { qw + NSAIDs }\end{array}$ & $12 \mathrm{w}$ & (3) (4) (5) (6) \\
\hline Tian [16] & $30(7 / 23)$ & $30(6 / 24)$ & $61.37 \pm 10.43$ & $59.47 \pm 11.77$ & $\begin{array}{l}\mathrm{KXC} 0.3 \mathrm{~g} \\
\text { tid + control }\end{array}$ & LEF $20 \mathrm{mg}$ qd & $24 \mathrm{w}$ & (4) (5) (6) \\
\hline Liu et al. [18] & $50(4 / 46)$ & $30(2 / 28)$ & $36-62$ & $35-59$ & KXC $0.6 \mathrm{~g}$ tid & LEF $20 \mathrm{mg}$ qn & $12 \mathrm{w}$ & (4) (5) (6) (7) \\
\hline Lu [19] & $30(11 / 19)$ & $30(10 / 20)$ & $46.31 \pm 5.62$ & $47.50 \pm 5.71$ & KXC $0.6 \mathrm{~g}$ tid & MTX $10-15$ mg qw & $6 \mathrm{~m}$ & (3) (4) (5) \\
\hline Lin et al. [17] & $80(24 / 56)$ & $79(13 / 66)$ & $52.14 \pm 9.70$ & $48.62 \pm 13.01$ & KXC $0.3-0.6 \mathrm{~g}$ tid & MTX $10-15$ mg qw & $12 \mathrm{w}$ & (1) (2) (3) (4) (5) (6) (7) \\
\hline
\end{tabular}

Note. KXC: Kunxian Capsule; MTX: methotrexate; LEF: leflunomide; HCQ: hydroxychloroquine; (1) TJC: tender joint counts; (2) SJC: swollen joint counts; (3) DMS: duration of morning stiffness; (4) ESR: erythrocyte sedimentation rate; (5) CRP: C-reactive protein; (6) RF: rheumatoid factor; (7) CCP: anticyclic citrullinated peptide antibody. Values are mean \pm standard deviation (SD).

difference between the two groups $(P=0.16, \mathrm{MD}=-0.87$, 95\% CI: -2.08 to 0.34$)$. Three studies compared KXC plus DMARDs with DMARDs regarding TJC (involving 160 subjects), and there was also no significant difference between the two groups $(P=0.05, \mathrm{MD}=-1.29,95 \% \mathrm{CI}:-2.60$ to 0.02 ). However, the overall effect (involving 319 subjects) showed that KXC group was superior to the control group regarding lowering the TJC $(P=0.02, \mathrm{MD}=-1.07,95 \% \mathrm{CI}$ : -1.95 to -0.18$)$.

3.6.2. The Outcome of SJC. As shown in Figure 3, only one study compared KXC with DMARDs in terms of SJC (including 159 subjects), and there was no significant difference between the two groups $(P=0.78, \mathrm{MD}=-0.14$, 95\% CI: -1.11 to 0.83 ). Three studies compared KXC plus DMARDs with DMARDs regarding SJC (including 160 subjects) and there was a significant difference between the two groups $(P=0.02, \mathrm{MD}=-1.42,95 \% \mathrm{CI}:-2.57$ to -0.27 ). Moreover, the overall effect (involving 319 subjects) demonstrated that there was no significant difference between the two groups $(P=0.08, \mathrm{MD}=-0.67,95 \%$ CI: -1.42 to 0.07$)$.

3.6.3. The Outcome of DMS. As indicated in Figure 3, two studies compared KXC with DMARDs with regard to DMS (involving 219 subjects) and there was a significant difference between the two groups $(P=0.0003, \mathrm{MD}=-8.28,95 \%$ CI: -12.80 to -3.76$)$. Four studies compared KXC plus DMARDs with DMARDs regarding DMS (involving 232 subjects), and there was a significant difference between the two groups $(P=0.01, \mathrm{MD}=-12.19,95 \% \mathrm{CI}:-21.63$ to -2.75). Moreover, the overall effect (involving 451 subjects) illustrated that KXC group was superior to the control group regarding reducing the DMS $(P<0.0001, \mathrm{MD}=-9.01,95 \%$ CI: -13.08 to -4.93$)$.
3.6.4. The Outcome of ESR. As illustrated in Figure 3, three studies compared KXC with DMARDs in terms of ESR (involving 299 subjects), and there was a significant difference between the two groups $(P<0.00001, \mathrm{MD}=-4.66$, 95\% CI: -6.35 to -2.97$)$. Six studies compared KXC plus DMARDs with DMARDs regarding ESR (involving 448 subjects), and there was a significant difference between the two groups $(P<0.00001, \mathrm{MD}=-7.68,95 \% \mathrm{CI}:-11.01$ to -4.35). Meanwhile, the overall effect (involving 747 subjects) showed that KXC group was superior to the control group regarding decreasing the ESR $(P<0.00001, \mathrm{MD}=-5.27$, 95\% CI: -6.78 to -3.77$)$.

3.6.5. The Outcome of CRP. As shown in Figure 3, three studies compared KXC with DMARDs in terms of CRP (involving 299 subjects), and there was a significant difference between the two groups $(P<0.00001, \mathrm{MD}=-7.29$, 95\% CI: -8.63 to -5.96$)$. Six studies compared KXC plus DMARDs with DMARDs regarding CRP (involving 448 subjects), and there was a significant difference between the two groups $(P=0.002, \mathrm{MD}=-3.98,95 \% \mathrm{CI}:-6.52$ to -1.43$)$. Meanwhile, the overall effect (involving 747 subjects) showed that KXC group was superior to the control group regarding declining $\mathrm{CRP}(P<0.0001, \mathrm{MD}=-5.04,95 \% \mathrm{CI}$ : -7.28 to -2.80$)$.

3.6.6. The Outcome of RF. As illustrated in Figure 3, two studies compared KXC with DMARDs in terms of RF (including 239 subjects), and there was no significant difference between the two groups $(P=0.51, \mathrm{MD}=51.28,95 \%$ CI: -101.94 to 204.50$)$. Five studies compared KXC plus DMARDs with DMARDs regarding RF (involving 408 subjects), and there was a significant difference between the two groups $(P=0.0005, \mathrm{MD}=-19.07,95 \% \mathrm{CI}:-29.76$ to -8.39 ). However, the overall effect (involving 647 subjects) 

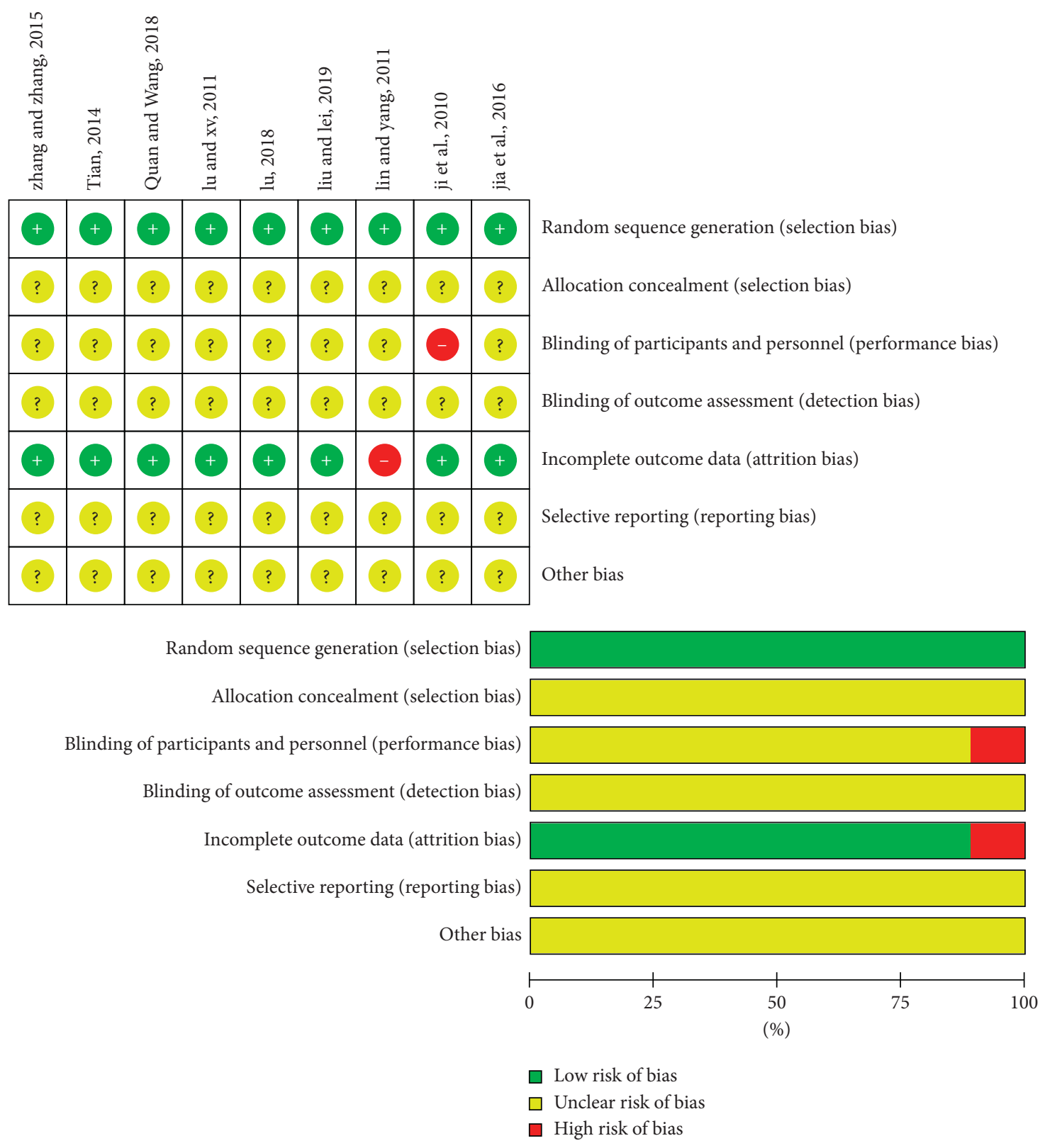

Figure 2: Risk of bias of the included studies.

showed that there was no significant difference between the two groups ( $P=0.96, \mathrm{MD}=-0.70,95 \% \mathrm{CI}:-31.79$ to 30.40$)$.

3.6.7. The Outcome of CCP. As depicted in Figure 3, two studies compared KXC with DMARDs in terms of CCP (involving 239 subjects), and there was no significant difference between the two groups $(P=0.27, \mathrm{MD}=-49.16$, $95 \%$ CI: -136.46 to 38.15$)$. Two studies compared KXC plus DMARDs with DMARDs in terms of CCP (involving 216 subjects), and there was no significant difference between the two groups $(P=0.24, \mathrm{MD}=-74.53,95 \% \mathrm{CI}:-198.85$ to 49.79). Meanwhile, the overall effect (involving 455 subjects) showed that there was no significant difference between the two groups $(P=0.05, \mathrm{MD}=-61.80,95 \% \mathrm{CI}:-124.85$ to 1.25).

3.7. The Adverse Events of KXC. As illustrated in Table 2, the adverse events were reported in six trials (involving $97 \mathrm{pa}-$ tients), 44 patients in KXC group, and 53 patients in the control group, respectively. The frequent adverse events were gastrointestinal discomfort and abnormal liver function.

\section{Discussion}

Complementary and alternative medicine has been extensively employed in the treatment of inflammatory arthritis 


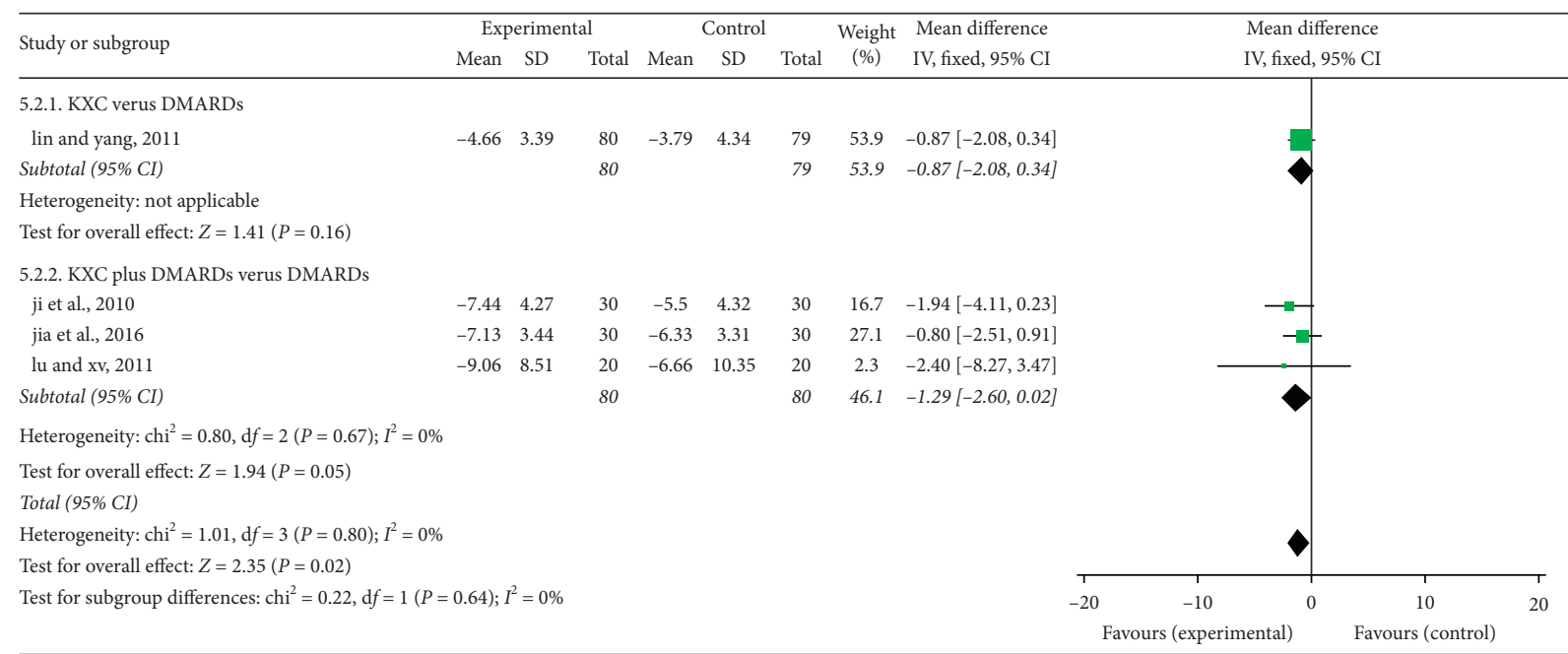

(a)

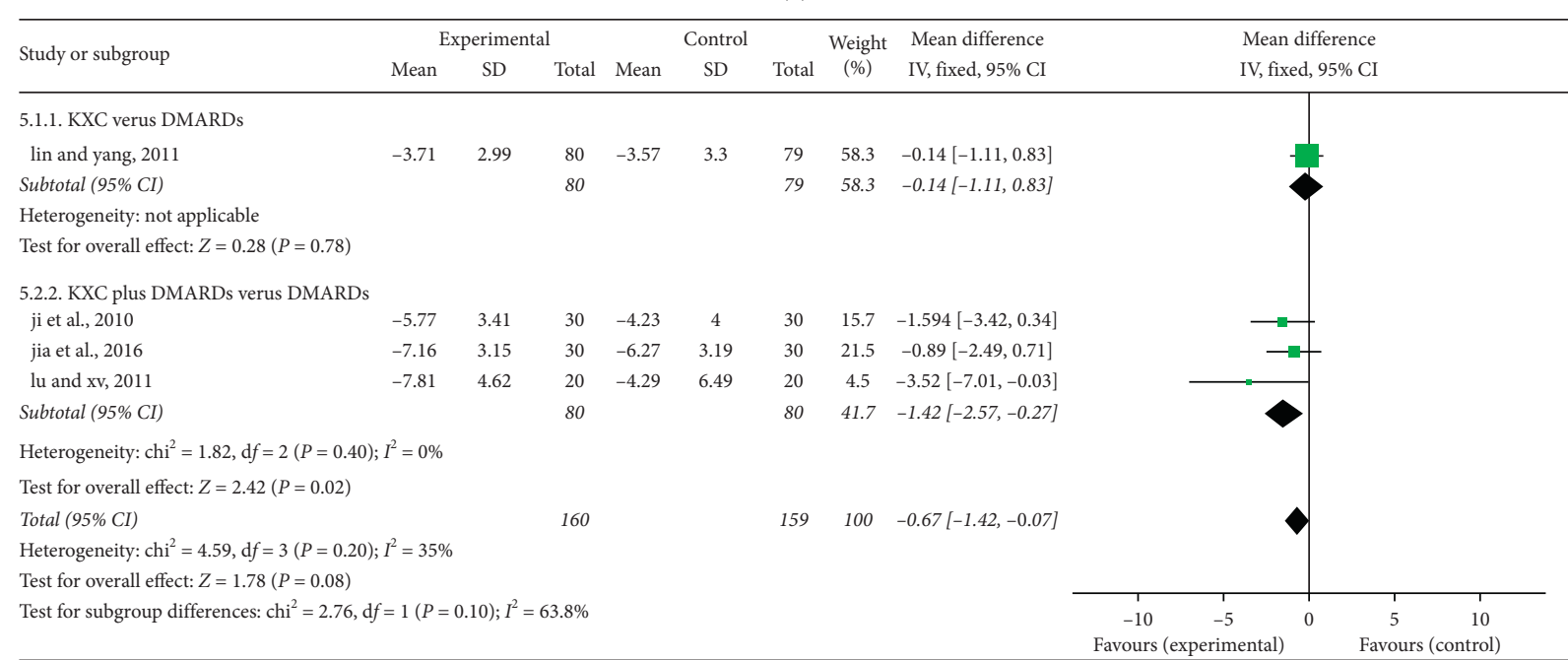

(b)

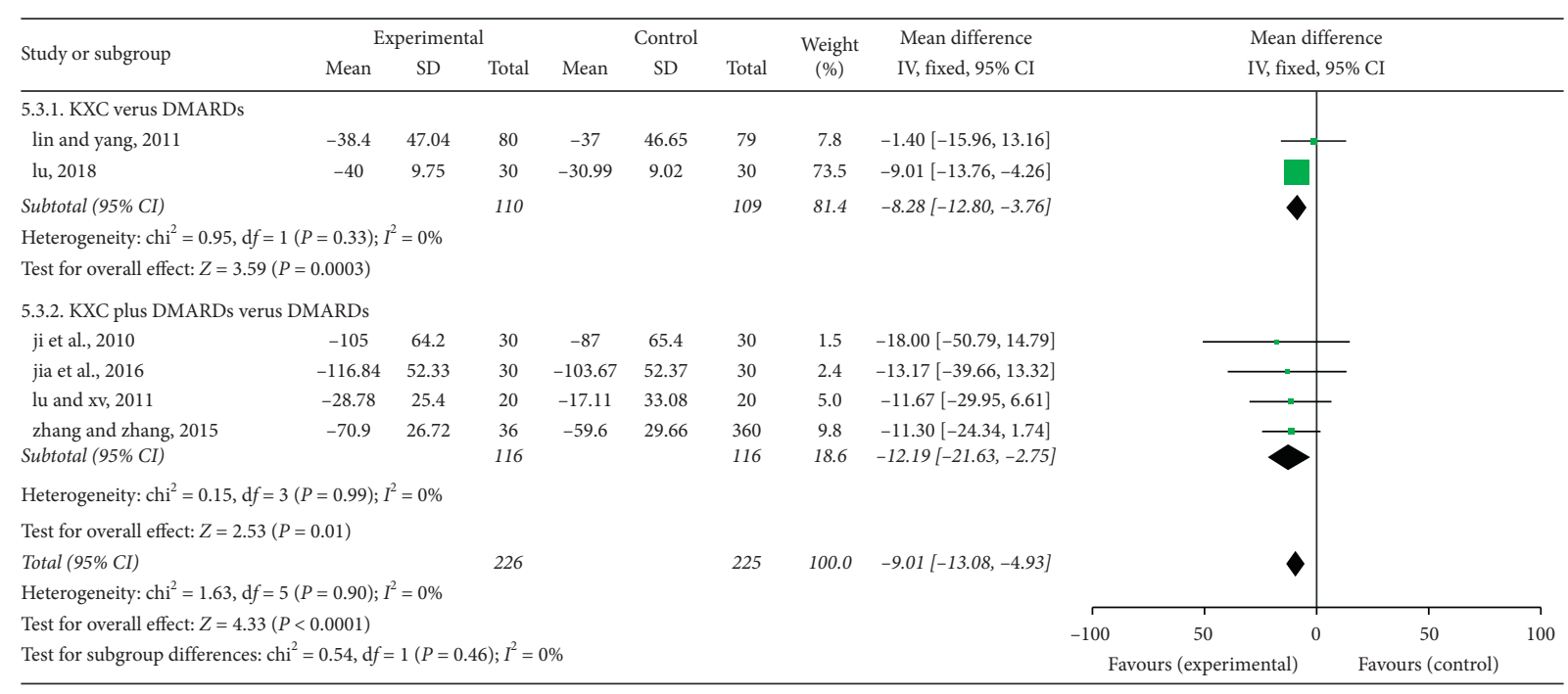

(c)

FIgURE 3: Continued. 


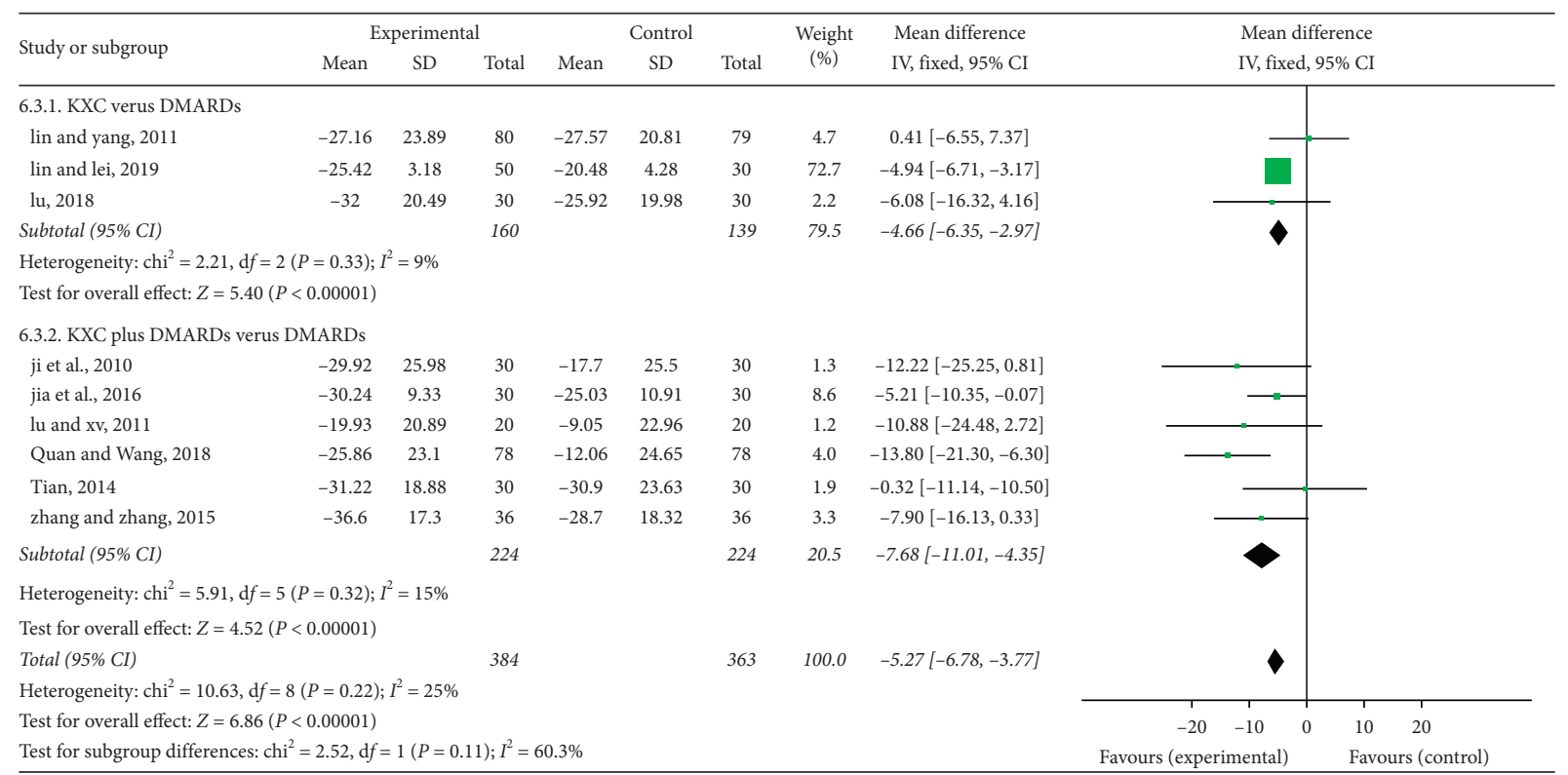

(d)

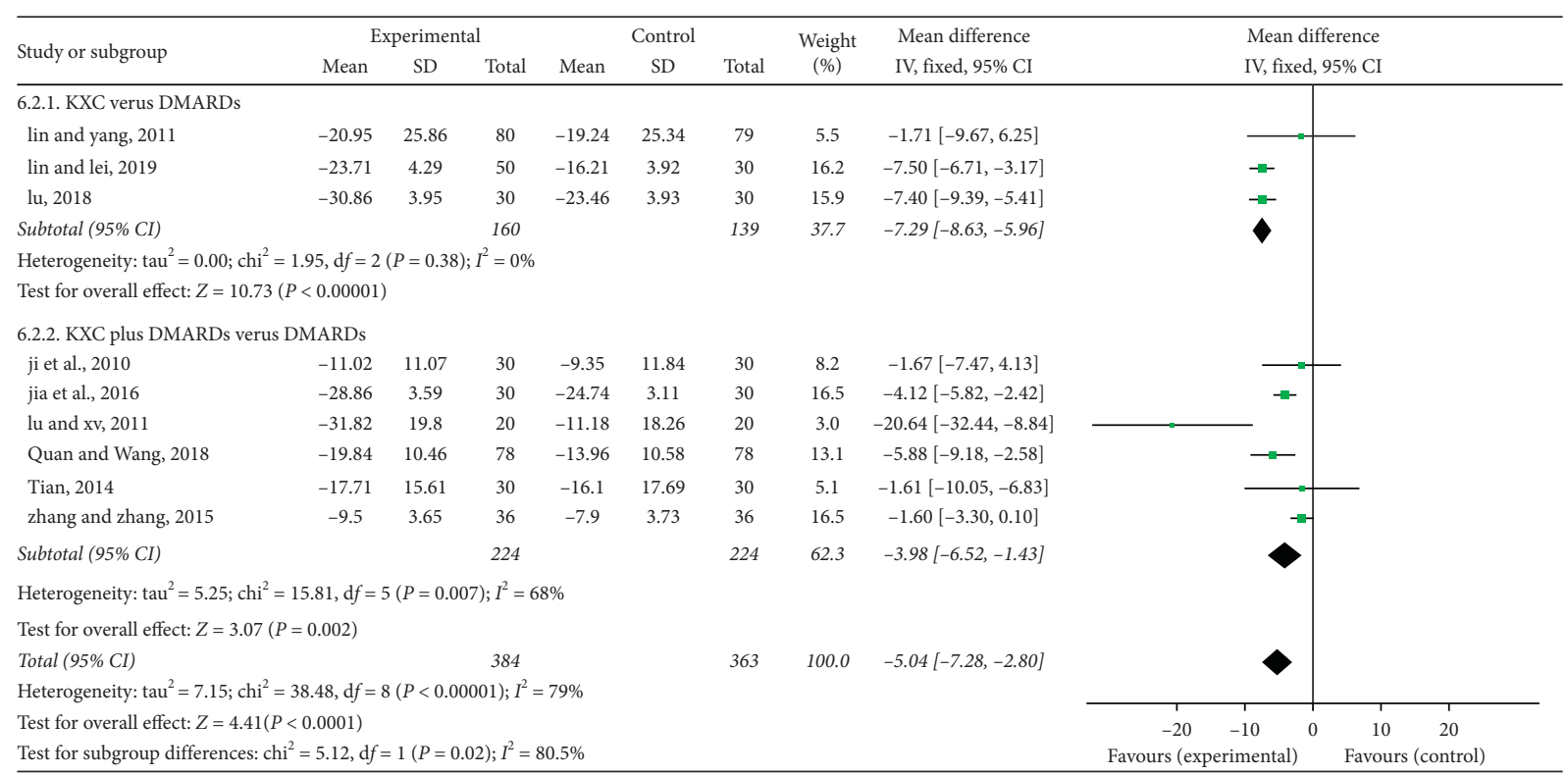

(e)

Figure 3: Continued. 


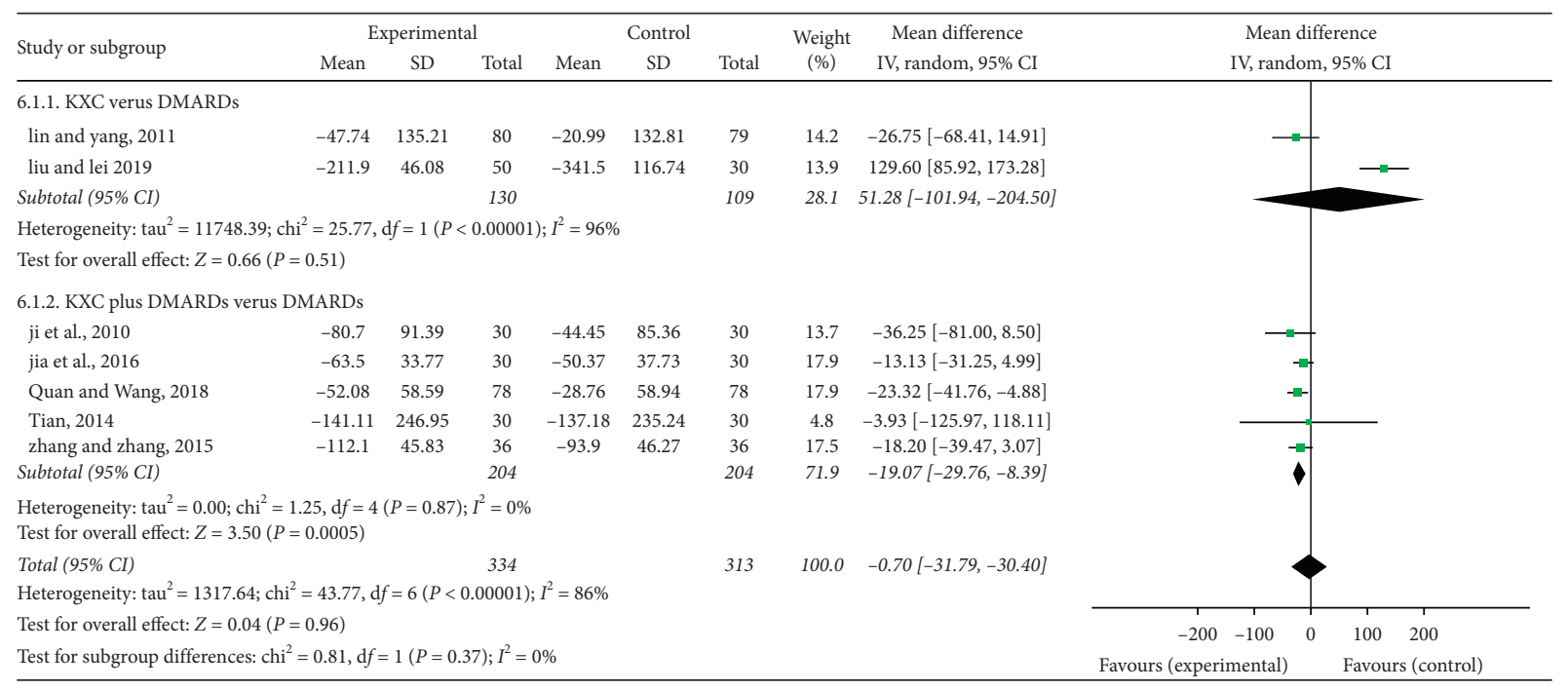

(f)

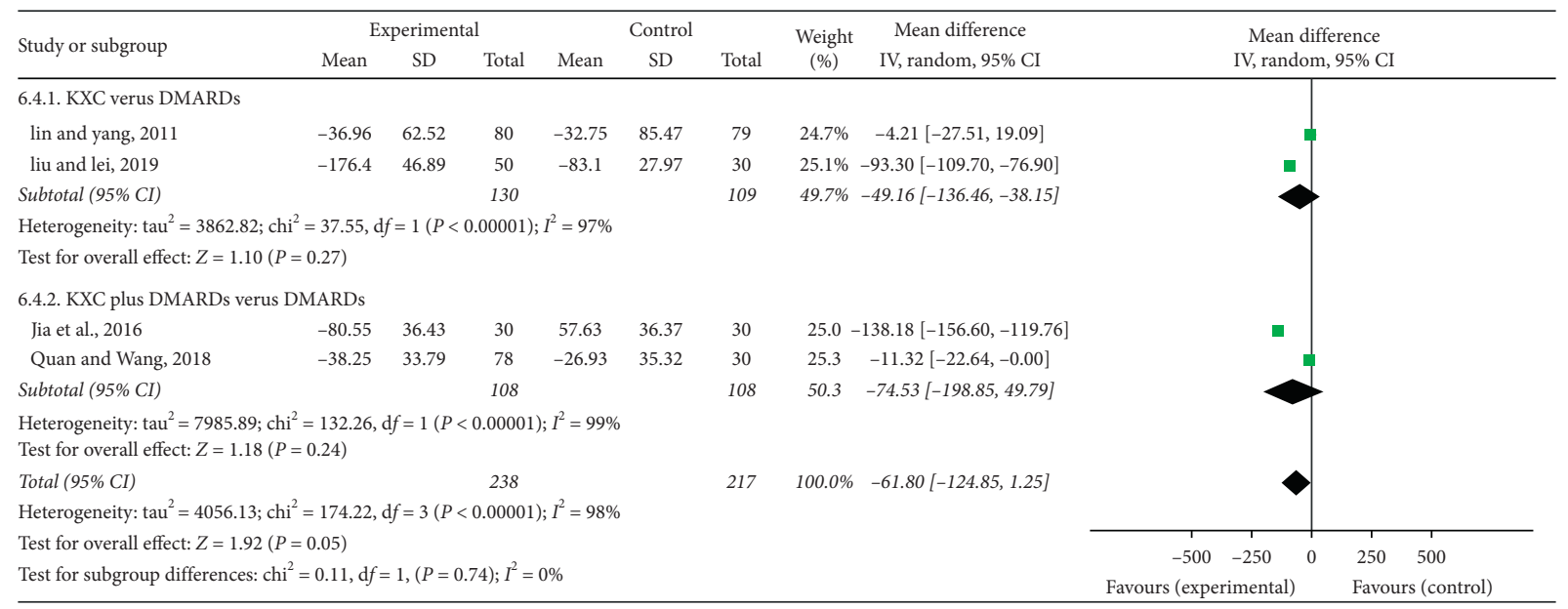

(g)

FIgURE 3: Forest plots for the outcomes. TJC: tender joint count; SJC: swollen joint count; DMS: duration of morning stiffness; ESR: erythrocyte sedimentation rate; CRP: C-reactive protein; RF: rheumatoid factor; and CCP: anticyclic citrullinated peptide.

[25]. KXC consists of four individual herbs which adhere to the compatibility principle of traditional Chinese medicine: Tripterygium hypoglaucum, Herba Epimedii, Fructus Lycii, and Semen Cuscuta. The main component of the KXC is TWHF, which derives from Tripterygium hypoglaucum $[26,27]$. Herba Epimedii was widely used in the clinic to enhance bone fracture healing [28]. Fructus Lycii had many pharmacological and biological activities, such as anti-inflammation, antioxidation, antiapoptosis, immune regulation [29]. Semen Cuscuta had a protective effect on the reproductive system [30]. In a word, $\mathrm{KXC}$, as an upgrade of TWHF, served as strengthening the therapeutic effect and weakening the side effects of TWHF [31-34].

There were two meta-analyses of KXC in the treatment of RA [35, 36]. Nonetheless, no review protocol was registered. However, one of the meta-analysis lacked data and forest plots [35]. The other meta-analysis did not have strict inclusion criteria [36]. Our analysis included more samples, performed rigorously screening, and had two subgroup analyses.

The results demonstrated that $\mathrm{KXC}$ had significant effects in alleviating DMS, ESR, and CRP, whether in two different subgroups or as a whole. These results were consistent with previous meta-analyses $[35,36]$. Moreover, TJC was reduced in KXC group, which was similar to the previous meta-analysis [35].

In addition, the study also demonstrated that $\mathrm{KXC}$ plus DMARDs group could reduce SJC and RF, but KXC alone or the overall effect of KXC did not lower SJC and RF. However, the previous analyses suggested that KXC could reduce SJC and RF [36]. We considered that the number of RCTs treated with KXC alone is small, which could lead to the difference.

Our study indicated that $\mathrm{KXC}$ had no effect on CCP, and previous meta-analyses did not analyze the outcome CCP. The CCP is a diagnostic parameter that cannot reflect disease activity; thereby KXC could not reduce CCP. 


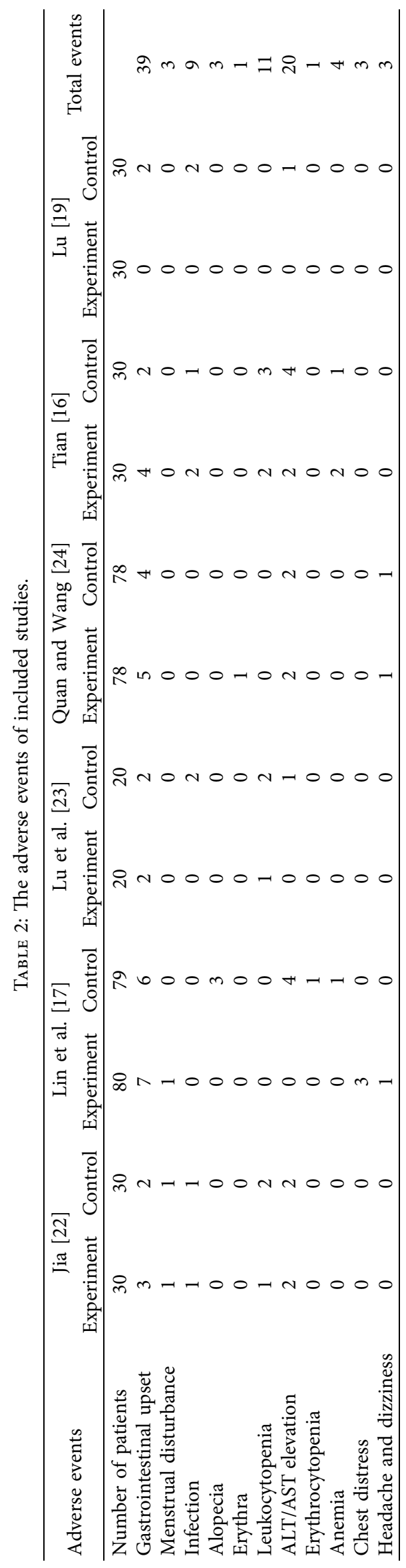


The mechanisms of KXC in treating RA have been explored in vitro and in vivo experiments. Previous research showed that KXC had immunosuppressive, anti-inflammatory, and analgesic effects [37]. Animal experiments demonstrated that KXC downregulated the expression of IL8 and $\gamma$ IP-10 mRNA, reduced synovial inflammation, alleviated the formation of pannus, decreased joint damage and fibrous tissue proliferation, and promoted tissue repair in the synovium of rats with collagen induced arthritis [38-40]. Additionally, triptolide influenced CD4+ and CD8+ cells distribution in Peyer's patch of DA rats with collagen induced arthritis [41].

However, some limitations of this systematic review should be mentioned. First, all the included trials were conducted in China, which inferred a high risk of selection bias. The results could not be applied to other regions in the world. Second, the majority of the studies published in Chinese were of poor quality. Only one multicenter RCT was confirmed. Third, the limited number of trials (from two to six) and participants included in each subgroup obscured the supportive evidence of KXC for RA. Fourth, the heterogeneity included in each subgroup was also obvious, especially in the outcomes CCP and RF. We consider that the differences in the quality of reports, intervention methods, doses, and duration of treatment lead to heterogeneity. Finally, the most important outcomes (ACR 20, ACR 50) were not reported except for two studies [17, 22]. Considering this, all the results should be carefully interpreted.

\section{Conclusion}

In conclusion, KXC is effective in the treatment of RA through lowering TJC, DMS, ESR, and CRP. KXC may function as anti-inflammation as well as immunosuppression. In a word, KXC, as an upgrade of TWHF, serves as enhancing the efficacy and weakening the side effects of TWHF. KXC, as a novel "phytoimmunosuppressant," is promising in the treatment of RA and other autoimmune diseases. Considering the low quality of the included trials, more well-designed RCTs are needed before we can recommend $\mathrm{KXC}$ to replace or combine with conventional therapies.

\section{Data Availability}

The data used to support the findings of this study are available from the corresponding author upon request.

\section{Conflicts of Interest}

All authors state that there are no conflicts of interest.

\section{Acknowledgments}

The authors would like to thank the National Natural Science Foundation of China for its financial support (81701601).

\section{References}

[1] D. L. Scott, F. Wolfe, and T. W. Huizinga, "Rheumatoid arthritis," The Lancet, vol. 376, no. 9746, pp. 1094-1108, 2010.

[2] G. S. Firestein, "Evolving concepts of rheumatoid arthritis," Nature, vol. 423, no. 6937, pp. 356-361, 2003.

[3] S. Safiri, A. A. Kolahi, D. Hoy et al., "Global, regional and national burden of rheumatoid arthritis 1990-2017: a systematic analysis of the global burden of disease study 2017," Annals of the Rheumatic Diseases, vol. 78, no. 11, pp. 14631471, 2019.

[4] R. W. Adeline and C. Arnaud, "Controversies in rheumatoid arthritis glucocorticoid therapy," Joint Bone Spine, vol. 85, no. 4, pp. 417-422, 2018.

[5] J. R. O’Dell, T. R. Mikuls, T. H. Taylor et al., "Therapies for active rheumatoid arthritis after methotrexate failure," New England Journal of Medicine, vol. 369, no. 4, pp. 307-318, 2013.

[6] S. Shankar and R. Handa, "Biological agents in rheumatoid arthritis," Journal of Postgraduate Medicine, vol. 50, no. 4, pp. 293-299, 2004.

[7] J. S. Smolen, D. Aletaha, M. Koeller, M. H. Weisman, and P. Emery, "New therapies for treatment of rheumatoid arthritis," Lancet, vol. 370, no. 9602, pp. 1861-1874, 2007.

[8] D. E. Furst, F. C. Breedveld, J. R. Kalden et al., "Updated consensus statement on biological agents for the treatment of rheumatoid arthritis and other rheumatic diseases (May 2002)," Annals of the Rheumatic Diseases, vol. 61, no. Suppl 2, pp. 2-7, 2002.

[9] X. Wang, Y. Zu, L. Huang et al., "Treatment of rheumatoid arthritis with combination of methotrexate and Tripterygium wilfordii: a meta-analysis," Life Sciences, vol. 171, pp. 45-50, 2017.

[10] Y. Liu, S. Tu, W. Gao et al., "Extracts of Tripterygium wilfordii Hook F in the treatment of rheumatoid arthritis: a systemic review and meta-analysis of randomised controlled trials," Evidence-Based Complementary and Alternative Medicine, vol. 2013, Article ID 410793, 11 pages, 2013.

[11] Y.-J. Zeng, H.-x. Kong, and S.-M. Li, "Clinical efficacy and safety of Kunxian capsule in the treatment of proteinuria in chronic kidney disease," Journal of New Chinese Medicine, vol. 46, no. 7, pp. 74-76, 2014.

[12] C.-P. Jia and P. Liu, "Research progress of kunxian capsule in the treatment of rheumatoid arthritis," World Latest Medicine Information, vol. 16, pp. 25-26, 2016.

[13] Z.-R. Jiang, M.-L. Gao, and Y.-Y. Liu, "Clinical observation of kunxian capsule in the treatment of active rheumatoid arthritis," Guangming Journal of Chinese Medicine, vol. 30, no. 2, pp. 279-281, 2015.

[14] T.-Y. Fang, "Clinical observation on 40 cases of rheumatoid arthritis treated with kunxian capsule," China's Naturopathy, vol. 25, no. 7, p. 63, 2017.

[15] D. Moher, A. Liberati, J. Tetzlaff, and D. G. Altman, "Preferred reporting items for systematic reviews and meta-analyses: the PRISMA statement,” BMJ, vol. 339, p. b2535, 2009.

[16] Y. Tian, Clinical Study of Kunxian Capsule in the Treatment of Active Rheumatoid Arthritis, Dalian Medical University, Dalian, China, 2014.

[17] C.-S. Lin, X.-Y. Yang, L. Dai et al., "Multicenter clinical study of kunxian capsule in the treatment of rheumatoid arthritis," Chinese Journal of Integrated Traditional And Western Medicine, vol. 31, no. 6, pp. 769-774, 2011. 
[18] Y.-M. Liu, Y.-M. Lei, B. Zhang, L.-P. Xia, J. Lu, and H. Shen, "Clinical study of kunxian capsule in the treatment of rheumatoid arthritis," Progress of Anatomical Sciences, vol. 25, no. 1, pp. 17-20, 2019.

[19] Y. Lu, "Curative effect analysis of kunxian capsule in the treatment of rheumatoid arthritis," China Journal of Pharmaceutical Economics, vol. 13, no. 8, pp. 78-80, 2018.

[20] Y.-L. Ji, Clinical Observation of Methotrexate Combined with Kunxian Capsule in the Treatment of Rheumatoid Arthritis of Kidney Deficiency and Cold Coagulation Type, Guangzhou University of Traditional Chinese Medicine, Guangzhou, China, 2010.

[21] J. Zhang and W.-C. Zhang, "Clinical observation on 36 cases of rheumatoid arthritis treated with kunxian capsule," Guiding Journal of Traditional Chinese Medicine, vol. 21, no. 11, pp. 69-70, 2015.

[22] C.-P. Jia, Clinical Observation of Kunxian Capsule Combined with Methotrexate in the Treatment of Rheumatoid Arthritis of Cold-Dampness Stasis Type, Hubei University of Traditional Chinese Medicine, Wuhan, China, 2016.

[23] J. Lu, Y.-Z. Xu, and D.-S. Tang, "Efficacy and safety of Kunxian capsule combined with methotrexate in the treatment of elderly patients with rheumatoid arthritis," Journal of Chinese Medicinal Materials, vol. 34, no. 5, pp. 835-837, 2011.

[24] Y. Quan and J. Wang, "Clinical study of Kunxian capsule combined with hydroxychloroquine sulfate in the treatment of rheumatoid arthritis," Drugs \& Clinic, vol. 33, no. 10, pp. 2638-2641, 2018.

[25] Y.-F. Liu, Y. Huang, C.-Y.-Z. Wen et al., "The effects of modified simiao decoction in the treatment of gouty arthritis: a systematic review and meta-analysis," Evid Based Complement Alternat Med, vol. 2017, Article ID 6037037, 12 pages, 2017.

[26] C.-Q. Xie, P. Zhou, X. Li, and J.-W. Chen, "Advances in chemical constituents, pharmacological activities and clinical application of kunming shanhaitang," Chinese Traditional and Herbal Drugs, vol. 46, no. 13, pp. 1996-2010, 2015.

[27] D.-P. Fan, Q.-Q. Guo, K. Zheng, Y.-K. Zhao, A.-P. Lv, and X.-J. He, "Effect of triptolide on expression of MIP-1a, eotaxin and MCP-1 in collagen induced arthritis rats," Modernization of Traditional Chinese Medicine and Materia Materia-World Science and Technology, vol. 18, no. 6, pp. 1027-1032, 2016.

[28] X. Huang, X. Wang, Y. Zhang et al., "Absorption and utilisation of epimedin $\mathrm{C}$ and icariin from Epimedii herba, and the regulatory mechanism via the BMP2/Runx2 signalling pathway," Biomedicine \& Pharmacotherapy, vol. 118, 2019.

[29] L. Lin, C. Guo, Z. Bing, and L. Feng, "Lycium barbarum polysaccharide induced apoptosis and inhibited proliferation in infantile hemangioma endothelial cells via down-regulation of PI3K/AKT signaling pathway," Bioscience Reports, vol. 39, no. 8, 2019.

[30] M. Mingsan, P. Mengfan, Z. Zhengwang, Y. Xiaoli, W. Zhenzhen, and L. Mengyan, "Effects of dodder total flavone on polycystic ovary syndrome rat models induced by DHEA combined HCG," Saudi Journal of Biological Sciences, vol. 26, no. 4, pp. 821-827, 2019.

[31] Y.-M. Xie, L.-L. Qin, X.-D. Yu, A.-D. Bao, and W.-L. Deng, “A comparative study on the effects of total flavonoids from radix drynariae, herba epimedii and cuscuta chinensis on osteoblasts culture in vitro," Chinese Journal of Information on Traditional Chinese Medicine, no. 7, pp. 22-24, 2005.

[32] J. Wang, J.-P. Li, Y.-W. Zhang, and S.-P. Hu, "Advances in pharmacology of epimedium," China Pharmaceuticals, vol. 18, no. 8, pp. 60-61, 2009.
[33] Y.-F. Zhu, "Overview of pharmacological action of fructus lycii," Zhejiang Journal of Integrated Traditional Chinese and Western Medicine, no. 5, pp. 322-323, 2005.

[34] W. Zhang, S.-H. Chen, and G.-Y. Lv, "Study on the correlation between the meridian tropism of efficacy and taste of Cuscuta chinensis and modern pharmacology," Lishizhen Medicine and Materia Medica Research, vol. 21, no. 4, pp. 808-811, 2010.

[35] H. Xu, "Effectiveness and safety of kunxian capsule in the treatment of rheumatoid arthritis," China Practical Journal of Medicine, vol. 43, no. 11, 2016.

[36] J. Zhou, W. Xiao, R. Wu, and X.-L. Shi, "Systematic evaluation of the efficacy and safety of kunxian capsule in the treatment of rheumatoid arthritis," Journal of Liaoning University of Traditional Chinese Medicine, vol. 18, no. 10, pp. 122-126, 2016.

[37] N. Zhang and W.-Y. Yi, "Progress in clinical application of kunxian capsule," Clinical Journal of Chinese Medicine, vol. 6, no. 7, pp. 147-148, 2014.

[38] C.-S. Lin, X.-D. Wang, Q. Xu et al., "Effect of kunxian capsule on the expression of IL- 8 and $\gamma$ IP-10 in synovial tissue of rats with collagen induced arthritis," Journal of New Chinese Medicine, vol. 44, no. 5, pp. 144-146, 2012.

[39] X.-D. Wang, Q. Xu, C.-S. Lin, Z.-H. Guo, Y.-B. Lin, and Z.-L. Li, "Effect of kunxian capsule on synovial membrane and serum $\gamma$ IP-10 in rats with induced arthritis," Journal of Liaoning University, vol. 14, no. 7, pp. 161-164, 2012.

[40] Q. Xu, C.-S. Lin, X.-D. Wang, Q.-P. Liu, L.-J. Liu, and Y.-B. Lin, "Effect of kidney-tonifying and rheumatism-dispelling therapy on synovial membrane and serum IL-8 in CIA rats," in Proceedings of the Tenth National Academic Conference on Rheumatism with Integrated Traditional Chinese and Western Medicine, vol. 2, Chengdu, Sichuan, China, 2012.

[41] C. Xiao, L. Zhao, Z. Liu et al., "The effect of triptolide on CD4+ and CD8+ cells in the peyer's patch of DA rats with collagen induced arthritis," Natural Product Research, vol. 23, no. 18, pp. 1699-1706, 2009. 